\title{
Lateral-compartment Osteophytes are not Associated With Lateral-compartment Cartilage Degeneration in Arthritic Varus Knees
}

\author{
Wenzel Waldstein MD, Maximilian F. Kasparek MD, Martin Faschingbauer MD, \\ Reinhard Windhager MD, PhD, Friedrich Boettner MD
}

Received: 19 July 2016/ Accepted: 31 October 2016/Published online: 9 November 2016

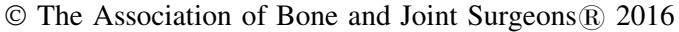

\begin{abstract}
Background Progression of arthritis in the lateral compartment is one of the main failure modes of unicompartmental knee arthroplasty (UKA). The decision regarding whether to perform a medial UKA sometimes is made based on whether lateral-compartment osteophytes are visible on plain radiographs obtained before surgery,
\end{abstract}

One of the authors (FB) certifies that he or a member of his immediate family, has or may receive payments or benefits, during the study period, an amount of less than USD 10,000 from Smith \& Nephew (Memphis, TN, USA), an amount of USD 10,000 to 100,000 from Ortho Development Corporation (Draper, UT, USA) outside the submitted work

The institution of one or more of the authors $(\mathrm{FB})$ has received funding from Smith \& Nephew (Memphis, TN, USA)

All ICMJE Conflict of Interest Forms for authors and Clinical Orthopaedics and Related Research ${ }^{\circledR}$ editors and board members are on file with the publication and can be viewed on request.

Each author certifies that his institution has approved the human protocol for this investigation, that all investigations were conducted in conformity with ethical principles of research and that informed consent for participation in the study was obtained.

This work was performed at the Hospital for Special Surgery, New York, NY, USA.

M. F. Kasparek, F. Boettner ( $\square)$

Adult Reconstruction \& Joint Replacement Division, Hospital for Special Surgery, 535 East 70th Street, New York, NY 10021, USA

e-mail: boettnerf@hss.edu

W. Waldstein, R. Windhager

Department of Orthopedic Surgery, Vienna General Hospital, Medical University of Vienna, Waehringer Guertel 18-20,

1090 Vienna, Austria

M. Faschingbauer

Department of Orthopedic Surgery, Ulm University Hospital, University of Ulm, Oberer Eselsberg 45, 89081 Ulm, Germany but it is not clear whether the presence of lateral-compartment osteophytes signifies that the cartilage in the lateral compartment is arthritic.

Questions/purposes (1) Is the presence of lateral compartment osteophytes associated with biomechanical properties of lateral-compartment cartilage, and (2) are osteophytes in the lateral compartment associated with particular histologic features of cartilage in the lateral compartment?

Methods Between May 2010 and January 2012, we performed 201 TKAs for varus osteoarthritis confirmed on standardized AP hip-to-ankle standing radiographs. All patients with a varus deformity were considered for this prospective study. During the enrollment period, 100 patients (101 knees) were not enrolled for this study because of declined consent or because they were unable to perform all required preoperative radiographic examinations. That left 84 patients (100 knees), of whom an additional 23 patients (27\%) were excluded because either radiographic or biomechanical data were missing. For final analysis, 61 patients (71 knees) were available. There were 29 males (48\%) and 32 females (52\%) with a mean age of 65 years (range, $49-89$ years). Their mean BMI was $26 \mathrm{~kg} /$ $\mathrm{m}^{2}$ (range, $17-47 \mathrm{~kg} / \mathrm{m}^{2}$ ). Lateral-compartment osteophytes were graded by two observers on AP standing knee radiographs based on a template of the Osteoarthritis Research Society International (OARSI) radiographic atlas. During surgery, osteochondral plugs were harvested from the lateral tibial plateau and the distal lateral femur for biomechanical and histologic assessments. The intrinsic material coefficients aggregate modulus $(\mathrm{Ha})$ and dynamic modulus (DM) were determined by applying a compressive load of $20 \mathrm{~g}$ for 1 hour. The histologic analysis was performed according to the qualitative osteoarthritis cartilage histopathology assessment system. The Mann-Whitney U 
test was performed to compare the distribution of variables. Power analysis was performed for the Mann-Whitney U test using an alpha of 0.05 , a power of $80 \%$, and a sample size of 71 resulting in a detectable effect size of 0.6. Owing to the limited sample size, only medium or large effects in changes of biomechanical properties can be excluded with adequate power.

Results Ha and DM were not different with the numbers available when comparing knees with osteophyte Grades 0 , 1 , and 2 on the lateral tibia. For Grade 3 tibial osteophytes $(\mathrm{n}=3)$, the lateral tibia cartilage showed low Ha $(0.39$ MPa; SD, 0.17 MPa) and low DM (2.85 MPa; SD, 2.12 $\mathrm{MPa}$ ). On the lateral femur, no differences of $\mathrm{Ha}$ and DM were observed with the numbers available between Grades 0 to 3 osteophytes. No differences with the numbers available in the OARSI histologic grades on the lateral tibia plateau and the distal lateral femur were observed between the different osteophyte grades.

Conclusions Lateral-compartment osteophytes are not associated with biomechanically weaker cartilage or with more-advanced histologic signs of degeneration of lateralcompartment cartilage in knees with varus arthritis. Given the small sample size of 71, the study was underpowered to detect small-to-modest decreases in biomechanical properties. Future studies with larger sample sizes are needed to confirm the current findings.

Clinical Relevance Factors other than the presence or absence of lateral-compartment osteophytes should be considered when evaluating patients with medial-compartment arthritis for medial UKA. Future studies are required to define the limitations of plain radiographs to rule out cartilage degeneration in the lateral compartment of varus knees.

\section{Introduction}

Careful patient selection is crucial to ensure excellent longterm results after unicompartmental knee arthroplasty (UKA) [9]. Lateral-compartment arthritis is considered a contraindication for a medial UKA [9], and progression of arthritis in the lateral compartment is one of its main failure modes [18]. According to Kellgren and Lawrence [11], osteoarthritis (OA) is defined by a definite osteophyte and possible narrowing of the joint space (as is seen in Kellgren-Lawrence Grade 2). Based on this threshold for arthritis, some surgeons believe that lateral-compartment osteophytes are a contraindication for medial UKA $[12,19,22]$.

Osteophyte formation is clearly associated with cartilage damage; however, osteophytes also may develop without cartilage damage owing to joint instability or lateral jointspace opening [20, 24]. In a recent publication,
Faschingbauer et al. [6] suggested that lateral-compartment osteophytes in varus $\mathrm{OA}$ of the knee are not associated with lower cartilage volume and diminished cartilage thickness in the lateral compartment. However, it remains unclear whether the presence of lateral-compartment osteophytes signifies that cartilage in the lateral compartment is arthritic.

The dataset used in this study was analyzed in earlier work [26-29]. A biomechanical cartilage evaluation of the Osteoarthritis Research Society International (OARSI) histologic assessment system was performed in one study [28]. The study showed that the OARSI histologic assessment system provides useful information regarding the biomechanical properties of cartilage [28]. Another study on the same dataset showed that an elevated white blood cell count in the synovial fluid of the knee is associated with inferior biomechanical cartilage properties [29]. In a radiographic study, the value of valgus stress radiographs in the workup of medial-compartment arthritis was analyzed. The study showed that valgus stress radiographs provide no added benefit in the assessment of lateralcompartment cartilage [26]. Previous work has contributed to a more-accurate preoperative evaluation of lateralcompartment cartilage in patients considered for medial UKA. However, an association of lateral-compartment osteophytes with cartilage degeneration in the lateral compartment has not been investigated to our knowledge, and we used this same dataset to explore this new question.

The current study assessed the presence of lateralcompartment osteophytes, and correlated newly collected data on lateral-compartment osteophytes with previously collected data on the histologic and biomechanical properties of cartilage in the lateral compartment. We asked the following research questions for osteoarthritic knees with varus alignment: (1) Is the presence of lateral-compartment osteophytes associated with biomechanical properties of lateral-compartment cartilage, and (2) are osteophytes in the lateral compartment associated with particular histologic features of cartilage in the lateral compartment?

\section{Patients and Methods}

The current study is a retrospective analysis of a dataset collected for a previous prospective study [28]. Between May 2010 and January 2012, we performed 201 TKAs for varus OA confirmed on standardized AP hip-to-ankle standing radiographs. All patients with a varus deformity were considered for this prospective study. During the enrollment period, 100 patients (101 knees) were not enrolled for this study because of declined consent or because they were unable to perform all required preoperative radiographic examinations. That left 84 patients 
(100 knees), of whom an additional 23 patients (27\%) were excluded because either radiographic or biomechanical data were missing. For final analysis, 61 patients (71 knees) were available. There were 29 males (48\%) and 32 females $(52 \%)$ with a mean age of 65 years (range, 49-89 years). Their mean BMI was $26 \mathrm{~kg} / \mathrm{m}^{2}$ (range, $17-47 \mathrm{~kg} / \mathrm{m}^{2}$ ).

All patients received preoperative standardized AP hipto-ankle standing radiographs and AP standing knee radiographs. All images were stored in a generic Digital Imaging and Communications in Medicine (DICOM) format. The study was approved by the institutional review board and all participants signed an informed consent.

The grading and all measurements were performed on a picture archiving and communication system (PACS) with commercial planning software (Sectra IDS7 ${ }^{\mathrm{TM}}$; Sectra, Linköping, Sweden). The angle between the femoral mechanical axis and the tibial mechanical axis was defined as the hip-knee-ankle angle on AP hip-to-ankle standing radiographs [15-17]. In the current study, the mean mechanical alignment of the knee was $9^{\circ}\left(\mathrm{SD}, 4^{\circ}\right.$; range, $1^{\circ}-19^{\circ}$ ) varus. Lateral-compartment osteophytes were graded on AP standing knee radiographs. The revised OARSI radiographic atlas is a well-accepted open-access tool for grading hand, hip, and knee radiographs [1]. The OARSI radiographic atlas was used as a template for this study. Osteophytes were grouped in Grade 0 (no osteophytes), Grade 1 (mild changes), Grade 2 (moderate changes), and Grade 3 (severe changes), respectively.

The methods pertaining to the biomechanical and histologic tissue analyses were described in previous studies $[28,29]$. Briefly, a compressive load of $20 \mathrm{~g}$ was applied at a rate of $5 \mathrm{~g}$ per second and held for 1 hour to determine the intrinsic material coefficients aggregate modulus (Ha) and dynamic modulus (DM), respectively. The Ha is a measure of the stiffness of the cartilage at equilibrium when all fluid flow has ceased. The DM is calculated by determining stress and strain during the initial phase of biomechanical testing. The histologic analysis was performed according to the OA cartilage histopathology assessment system
(OARSI histologic system) [21]. Biomechanical cartilage properties for different OARSI histologic grades have been described [28]. Intra- and interobserver reliabilities for 20 randomly selected AP standing radiographs were evaluated by two independent observers (WW, JBM) using singlemeasure intraclass correlation coefficients (ICC). Excellent intraobserver ICC was seen for grading of lateral tibial osteophytes (0.967) and lateral femoral osteophytes (0.964). Interobserver ICC also showed similar correlations: lateral tibial osteophytes (0.904) and lateral femoral osteophytes (0.947). An excellent intraobserver ICC for the histologic analysis was described before [28].

The majority of knees (92\%; 65 of 71) had evidence of at least one definite osteophyte in the lateral-compartment (lateral tibia plateau (Table 1) and/or lateral femoral condyle (Table 2).

\section{Statistical Analysis}

The distributions of all variables were examined in exploratory data analyses, and tested for normality using the Kolmogorov-Smirnov test. The Mann-Whitney U test was performed to compare the distribution of variables as not all parameters met the criteria for a normal distribution. The Spearman rank correlation $\left(r_{s}\right)$ was used for nonparametric correlations. Simple linear regression was performed to determine whether age, sex, and the degree of varus deformity are predictor variables for the presence and size of lateral-compartment osteophytes. A two-way mixed model with $95 \%$ CIs was used for calculation of the ICC. Single measures are given for intraobserver calculations. Probability values less than 0.05 were considered significant.

Power analysis was performed in G-power $\left(\mathrm{G}^{*}\right.$ Power Version 3.1.2; University of Kiel, Kiel, Germany) for the Mann-Whitney $U$ test using an alpha of 0.05 , a power of $80 \%$, and a sample size of 71 resulting in a detectable effect size (ES) of 0.6. Owing to the limited sample size, only medium and large effects in changes of biomechanical

Table 1. Association of lateral tibial osteophytes and microscopic cartilage assessment on the lateral tibia plateau

\begin{tabular}{llllll}
\hline Histology & \multicolumn{2}{l}{ Osteophytes } & & \\
\cline { 2 - 6 } OARSI grade & Grade 0 & Grade 1 & Grade 2 & Grade 3 & Total (number of knees) \\
\hline 0 & 1 & 4 & 1 & 0 & $6(8 \%)$ \\
1 & 1 & 7 & 5 & 1 & $14(20 \%)$ \\
2 & 6 & 20 & 4 & 0 & $30(42 \%)$ \\
3 & 1 & 11 & 0 & 0 & 0 \\
4 & 0 & 0 & 1 & 1 & $4(24 \%)$ \\
5 & 1 & 1 & $15(21 \%)$ & $3(4 \%)$ & $71(100 \%)$ \\
\hline
\end{tabular}

OARSI = Osteoarthritis Research Society International. 
Table 2. Association of lateral femoral osteophytes and microscopic cartilage assessment on the distal lateral femur

\begin{tabular}{|c|c|c|c|c|c|}
\hline \multirow{2}{*}{$\begin{array}{l}\text { Histology } \\
\text { OARSI grade }\end{array}$} & \multicolumn{4}{|c|}{ Osteophytes } & \multirow[b]{2}{*}{ Total (number of knees) } \\
\hline & Grade 0 & Grade 1 & Grade 2 & Grade 3 & \\
\hline 0 & 14 & 15 & 4 & 3 & $36(51 \%)$ \\
\hline 1 & 6 & 9 & 4 & 4 & $23(32 \%)$ \\
\hline 2 & 1 & 4 & 0 & 2 & $7(10 \%)$ \\
\hline 3 & 0 & 1 & 0 & 2 & $3(4 \%)$ \\
\hline 4 & 2 & 0 & 0 & 0 & $2(3 \%)$ \\
\hline 5 & 0 & 0 & 0 & 0 & 0 \\
\hline Total (number of knees) & $23(32 \%)$ & $29(41 \%)$ & $8(11 \%)$ & $11(16 \%)$ & $71(100 \%)$ \\
\hline
\end{tabular}

OARSI = Osteoarthritis Research Society International.

properties can be excluded with adequate power. Because of the relatively small sample size, the used test is underpowered to detect smaller effects. Means and SD units were used to calculate the standardized mean difference (SMD). Owing to the low number of knees with Grade 3 osteophytes on the tibia $(n=3)$, no calculations of statistical significance were performed for Grade 3 tibial osteophytes. Statistical tests were performed using SPSS Version 16.0 software for Windows (SPSS Inc, Chicago, IL, USA).

\section{Results}

Lateral Tibial Biomechanical Cartilage Properties

With the numbers available, diminished biomechanical properties of cartilage on the lateral tibia plateau were not associated with the presence of osteophytes on the lateral tibia. Comparison of aggregate modulus of cartilage on the lateral tibia between osteophyte Grades 0 and 1 showed that mean Ha (0.54 MPa; SD, 0.21) in osteophyte Grade 0 knees $(\mathrm{n}=10)$ was not different $(\mathrm{p}=0.838 ; \mathrm{ES}, 0.20)$ from mean $\mathrm{Ha}$ (0.59 MPa; SD, 0.26) in osteophyte Grade 1 knees $(n=43)$. Similarly, mean DM was not different ( $\mathrm{p}=0.865$; ES, 0.04) between osteophyte Grade 0 knees $(\mathrm{n}=10 ; 3.57 \mathrm{MP}$; SD, $1.90)$ and osteophyte Grade 1 knees $(n=43 ; 3.66 \mathrm{MPa}$; SD, 2.29). Mean Ha in tibial osteophyte Grade 1 knees $(0.59$ $\mathrm{MPa}$; SD, 0.26) was even lower ( $\mathrm{p}=0.035$; $\mathrm{ES}, 0.60)$ than mean Ha in tibial osteophyte Grade 2 knees $(0.74 \mathrm{MPa}$; SD, 0.24). Mean DM in tibial osteophyte Grade 1 knees (3.66 $\mathrm{MPa}$; SD, 2.29) was not different ( $\mathrm{p}=0.065$; ES, 0.50) compared with mean DM in tibial osteophyte Grade 2 knees (4.83 MPa; SD, 2.36). Mean Ha in knees with Grade 3 tibial osteophytes $(\mathrm{n}=3)$ was $0.39 \mathrm{MPa}(\mathrm{SD}, 0.17 \mathrm{MPa})$, and mean DM was $2.85 \mathrm{MPa}$ (SD, 2.12 MPa), respectively. However, owing to the low sample size, no statistical analysis was performed for knees with Grade 3 tibial osteophytes.
Distal Lateral Femoral Biomechanical Cartilage Properties

Osteophytes on the lateral femur were not associated with changes in the biomechanical cartilage properties of the distal lateral femur with the numbers available. There was no difference ( $p=0.176$; ES, 0.44$)$ of mean Ha in osteophyte Grade 0 knees $(\mathrm{n}=23 ; 1.18 \mathrm{MPa} ; \mathrm{SD}, 0.61)$ and osteophyte Grade 1 knees ( $\mathrm{n}=29 ; 0.95 \mathrm{MPa}$; SD, 0.45). Similarly, mean DM was not different $(\mathrm{p}=0.612$; ES, 0.33) between osteophyte Grade 0 knees $(\mathrm{n}=23 ; 6.43 \mathrm{MPa}$; SD, 3.70) and osteophyte Grade 1 knees ( $\mathrm{n}=29 ; 5.43 \mathrm{MPa}$; SD, 2.10). Between femoral osteophyte Grades 1 and 2, mean Ha in Grade 1 knees ( $=29 ; 0.95 \mathrm{MPa}$; SD, 0.45) was not different $(\mathrm{p}=0.871$; ES, 0.11) from mean Ha in Grade 2 knees ( $\mathrm{n}=8 ; 0.91 \mathrm{MPa}$; SD, 0.27). Similarly, mean DM in Grade 1 knees $(\mathrm{n}=29 ; 5.43 \mathrm{MPa}$; SD, 2.10) was not different $(\mathrm{p}=0.871$; ES, 0.08) from mean DM in Grade 2 knees $(\mathrm{n}=8 ; 5.57 \mathrm{MPa}$; SD, 1.46). Finally, there was no difference $(\mathrm{p}=0.409$; ES, 0.58) in mean Ha between Grade 2 knees ( $\mathrm{n}=8 ; 0.91 \mathrm{MPa} ; \mathrm{SD}, 0.27)$ and Grade 3 knees ( $\mathrm{n}=$ $11 ; 0.77 \mathrm{MPa}$; SD, 0.21). Mean DM also was not different $(\mathrm{p}=0.457$; ES, 0.46) between femoral osteophyte Grade 2 $(\mathrm{n}=8 ; 5.57 \mathrm{MPa} ; \mathrm{SD}, 1.46)$ and Grade $3(\mathrm{n}=11 ; 4.88$ $\mathrm{MPa}$; SD, 1.53).

\section{Cartilage Histology}

There was no relationship of osteophytes on the lateral tibia plateau and distal lateral femur and histologic features of cartilage in the lateral compartment. No difference of OARSI histologic grades on the lateral tibia plateau was observed between the different osteophyte grades with the numbers available (Table 1). Advanced degeneration on the lateral tibia plateau (OARSI histologic Grade 5, denudation) was evident in four knees. Of these, one knee had no osteophytes on the lateral tibia, one had a Grade 1 
osteophyte, one had a Grade 2 osteophyte, and one had a Grade 3 osteophyte, respectively. Similarly, no differences of OARSI histologic grades was observed with the numbers available between knees with Grades 0 to 3 osteophytes on the lateral femoral condyle (Table 2). Overall, two knees had an OARSI histologic Grade 4 cartilage lesion (erosion) on the distal lateral femur and these knees had no osteophytes on the lateral femur.

Age, sex, and degree of varus alignment did not explain the variability of tibial and femoral osteophytes. Sex $\left(R^{2}=\right.$ $0.032 ; \mathrm{p}=0.127)$ and the degree of varus alignment $\left(\mathrm{R}^{2}=\right.$ $0.003 ; \mathrm{p}=0.643$ ) were not associated with osteophyte grades on the lateral tibia. Increasing age was associated with smaller tibial osteophytes, however, there was considerable variability in this association $\left(\mathrm{R}^{2}=0.073 ; \mathrm{p}=\right.$ $0.022)$. Age $\left(R^{2}=0.015 ; p=0.309\right)$ and the degree of varus alignment $\left(R^{2}=0.032 ; p=0.143\right)$ were not associated with the presence of femoral osteophytes. Female gender was weakly associated with the presence of more femoral osteophytes $\left(\mathrm{R}^{2}=0.139 ; \mathrm{p}=0.001\right)$.

\section{Discussion}

One of the main causes of revision after medial UKA is progressive $\mathrm{OA}$ in the lateral compartment $[14,18]$. An accurate assessment of the quality of lateral-compartment cartilage therefore is essential when indicating UKA. However, it is currently unclear whether the presence of lateral-compartment osteophytes is associated with cartilage degeneration in the lateral compartment. Therefore, we asked: (1) Is the presence of lateral-compartment osteophytes associated with biomechanical properties of lateral-compartment cartilage, and (2) are osteophytes in the lateral compartment associated with particular histologic features of cartilage in the lateral compartment?

Our study has the following limitations. First, the $8-\mathrm{mm}$ diameter osteochondral samples obtained in the study do not represent the entire lateral compartment. However, the osteochondral samples represent the biomechanical and histologic conditions of cartilage in the weightbearing area of the distal lateral femur and the center of the lateral tibia plateau, respectively. Second, no biochemical analysis of cartilage samples was performed. However, Ha in samples with no degeneration was within the range previously reported for normal human lateral-compartment cartilage [23]. Only one study reported the DM of human cartilage in 13 knees [13]. In that study, comparable however slightly higher values of DM were reported compared with those in the current study. Therefore considering the normal biomechanical and histologic properties, normal biochemical properties can be assumed. Third, no histologic analysis of the osteophytes was performed. Although this might have been an interesting addition to our study it should not affect its conclusion.

In the 1950s, Kellgren and Lawrence [11] developed a radiographic grading system for OA which despite its age is still widely used. The classification did not provide guidelines regarding how to interpret its different grades. This led to problems in the clear classifications of OA. Kellgren-Lawrence Grade 2 is the threshold for arthritis and is commonly defined as a definite osteophyte and possible narrowing of the joint space [10]. The Kellgren and Lawrence system has been criticized as it overemphasizes the presence of osteophytes [7]. Derek et al. [3], therefore, suggested removing tibial osteophytes as a scoring parameter. In the current study, a definite osteophyte was observed on either the lateral femur and/or the lateral tibia in $92 \%$ (65 of 71) of knees. Based on the original Kellgren-Lawrence criteria, lateral-compartment arthritis therefore should have been suspected in $92 \%$ of the knees. However, our study suggests that lateral-compartment osteophytes in knees with varus deformities are poorly associated with cartilage degeneration in the lateral compartment. There is only one published study available to the best of our knowledge, analyzing the influence of lateral knee osteophytes on AP radiographs on the quality of cartilage in the lateral compartment of varus knees based on data from the Osteoarthritis Initiative [6]. Similar to the results in our study, Faschingbauer et al. [6] showed that volume and thickness of cartilage in the lateral compartment were not influenced by the presence of lateral-compartment osteophytes in varus $\mathrm{OA}$ of the knee.

Lateral osteophytes, observed on AP knee radiographs, were not associated with histologic cartilage degeneration in the lateral compartment. Osteophyte grades did not correlate with OARSI histologic grades. Consequently, there were a substantial number of knees with advanced cartilage degeneration, but with no or only minimal osteophytes observed on plain radiographs. These findings further illustrate that the Kellgren-Lawrence grading scale does not take the condition of cartilage appropriately into consideration. A standardized classification of $\mathrm{OA}$ is essential for clinical practice and arthritis research. Felson et al. [7] therefore modified Kellgren-Lawrence Grade 2 to require osteophytes and definite joint-space narrowing to be present for the diagnosis of OA. Such a modification might improve the sensitivity to detect OA; however, this only can be used with caution in the lateral compartment in varus OA as medial cartilage loss results in varus alignment and unloads (widens) the lateral joint space. Considering joint-space narrowing and the development of lateral-compartment osteophytes in the diagnosis of lateralcompartment OA therefore seems to be of limited value to 
select patients for a medial UKA. An adequate and reliable assessment of the lateral compartment remains challenging despite new insights regarding factors associated with lateral-compartment cartilage deterioration [28, 29]. The current study is not the first to show limitations of plain radiographs [2,25] and stress radiographs [26] to assess the lateral compartment in patients with varus deformities. Despite higher costs, MRI might be the only tool to improve preoperative evaluation of lateral-compartment cartilage in a noninvasive fashion. MRI allows for a high tissue contrast [4] and seems to be an excellent modality to assess the state of articular cartilage [5]. MRI is also an excellent tool to assess the integrity of the ACL [27]. This is important, as an intact ACL is an essential requirement for UKA [9] as higher UKA failure rates have been reported in patients with ACL insufficiency at the time of surgery [8]. However, if a surgeon is prepared intraoperatively to change the plan from a UKA to a TKA, the assessment of lateral-compartment cartilage and the integrity of the ACL may be made during surgery, obviating any need for advanced imaging before the procedure. The current study does not analyze whether lateral osteophytes are a contraindication for high tibial osteotomy or UKA. However, it provides supporting evidence that factors other than the presence or absence of lateralcompartment osteophytes should be considered when evaluating patients with medial-compartment arthritis for a high tibial osteotomy or a UKA.

Lateral-compartment osteophytes are not associated with biomechanically weaker cartilage or with more-advanced histologic signs of degeneration in the lateral compartment of knees with varus OA. Given the small sample size of 71 , the study was underpowered to detect small-to-modest decreases in biomechanical properties. Using the presence of a lateral osteophyte on radiographs to identify $\mathrm{OA}$ in the lateral compartment in varus knees will overestimate the presence of arthritis in that compartment. To improve patient selection for medial UKA, standards for a reliable and adequate assessment of the lateral compartment need to be developed. A better understanding of the limitations of plain radiographs to rule out cartilage degeneration in the lateral compartment of varus knees is required. Future studies with larger sample sizes are needed to confirm the current findings.

Acknowledgments We thank Giorgio Perino MD (Department of Pathology, Hospital for Special Surgery, New York, NY, USA) for the histologic analysis; Suzanne A. Maher PhD and Susannah L. Gilbert MS (Department of Biomechanics, Hospital for Special Surgery, New York, NY, USA) for help with the study design and analysis of biomechanical data; and Jad Bou Monsef MD (Department of Orthopedic Surgery, University of Illinois at Chicago, Chicago, IL, USA) for assistance with radiographic measurements.

\section{References}

1. Altman RD, Gold GE. Atlas of individual radiographic features in osteoarthritis, revised. Osteoarthritis Cartilage. 2007;15(suppl A):A1-56.

2. Brandt KD, Fife RS, Braunstein EM, Katz B. Radiographic grading of the severity of knee osteoarthritis: relation of the Kellgren and Lawrence grade to a grade based on joint space narrowing, and correlation with arthroscopic evidence of articular cartilage degeneration. Arthritis Rheum. 1991;34:1381-1386.

3. Derek T, Cooke V, Kelly BP, Harrison L, Mohamed G, Khan B. Radiographic grading for knee osteoarthritis. A revised scheme that relates to alignment and deformity. $J$ Rheumatol. 1999;26:641-644.

4. Disler DG, McCauley TR, Wirth CR, Fuchs MD. Detection of knee hyaline cartilage defects using fat-suppressed three-dimensional spoiled gradient-echo MR imaging: comparison with standard MR imaging and correlation with arthroscopy. AJR Am J Roentgenol. 1995;165:377-382.

5. Eckstein F, Guermazi A, Gold G, Duryea J, Hellio Le Graverand MP, Wirth W, Miller CG. Imaging of cartilage and bone: promises and pitfalls in clinical trials of osteoarthritis. Osteoarthritis Cartilage. 2014;22:1516-1532.

6. Faschingbauer M, Renner L, Waldstein W, Boettner F. Are lateral compartment osteophytes a predictor for lateral cartilage damage in varus osteoarthritic knees?: data from the Osteoarthritis Initiative. Bone Joint J. 2015;97:1634-1639.

7. Felson DT, Niu J, Guermazi A, Sack B, Aliabadi P. Defining radiographic incidence and progression of knee osteoarthritis: suggested modifications of the Kellgren and Lawrence scale. Ann Rheum Dis. 2011;70:1884-1886.

8. Goodfellow J, O'Connor J. The anterior cruciate ligament in knee arthroplasty: a risk-factor with unconstrained meniscal prostheses. Clin Orthop Relat Res. 1992;276:245-252.

9. Goodfellow JW, O'Connor J, Dodd CA, Murray DW. Unicompartmental Arthroplasty with the Oxford Knee. Oxford, UK: Oxford University Press; 2006.

10. Kellgren JH; Council for International Organizations of Medical Sciences. The Epidemiology of ChronicRrheumatism: A Symposium Organized by the Council for International Organizations of Medical Sciences. Symposium on Population Studies in Relation to Chronic Rheumatic Diseases. Oxford, UK: Blackwell; 1963.

11. Kellgren JH, Lawrence JS. Radiological assessment of osteoarthrosis. Ann Rheum Dis. 1957;16:494-502.

12. Kozinn SC, Scott R. Unicondylar knee arthroplasty. J Bone Joint Surg Am. 1989;71:145-150.

13. Kurkijarvi JE, Nissi MJ, Kiviranta I, Jurvelin JS, Nieminen MT. Delayed gadolinium-enhanced MRI of cartilage (dGEMRIC) and T2 characteristics of human knee articular cartilage: topographical variation and relationships to mechanical properties. Magn Reson Med. 2004;52:41-46.

14. Liddle AD, Judge A, Pandit H, Murray DW. Adverse outcomes after total and unicompartmental knee replacement in 101,330 matched patients: a study of data from the National Joint Registry for England and Wales. Lancet. 2014;384:1437-1445.

15. Marx RG, Grimm P, Lillemoe KA, Robertson CM, Ayeni OR, Lyman S, Bogner EA, Pavlov H. Reliability of lower extremity alignment measurement using radiographs and PACS. Knee Surg Sports Traumatol Arthrosc. 2011;19:1693-1698.

16. Merle C, Waldstein W, Pegg E, Streit MR, Gotterbarm T, Aldinger PR, Murray DW, Gill HS. Femoral offset is underestimated on anteroposterior radiographs of the pelvis but accurately assessed on anteroposterior radiographs of the hip. J Bone Joint Surg Br. 2012;94:477-482. 
17. Moreland JR, Bassett LW, Hanker GJ. Radiographic analysis of the axial alignment of the lower extremity. J Bone Joint Surg Am. 1987;69:745-749.

18. Murray DW, Liddle AD, Dodd CA, Pandit H. Unicompartmental knee arthroplasty: is the glass half full or half empty? Bone Joint J. 2015;97(10 suppl A):3-8.

19. Palumbo BT, Scott RD. Diagnosis and indications for treatment of unicompartmental arthritis. Clin Sports Med. 2014;33:11-21.

20. Pottenger LA, Phillips FM, Draganich LF. The effect of marginal osteophytes on reduction of varus-valgus instability in osteoarthritic knees. Arthritis Rheum. 1990;33:853-858.

21. Pritzker KP, Gay S, Jimenez SA, Ostergaard K, Pelletier JP, Revell PA, Salter D, van den Berg WB. Osteoarthritis cartilage histopathology: grading and staging. Osteoarthritis Cartilage. 2006;14:13-29.

22. Ritter MA, Faris PM, Thong AE, Davis KE, Meding JB, Berend ME. Intra-operative findings in varus osteoarthritis of the knee: an analysis of pre-operative alignment in potential candidates for unicompartmental arthroplasty. $J$ Bone Joint Surg $\mathrm{Br}$. 2004;86:43-47.

23. Treppo S, Koepp H, Quan EC, Cole AA, Kuettner KE, Grodzinsky AJ. Comparison of biomechanical and biochemical properties of cartilage from human knee and ankle pairs. J Orthop Res. 2000;18:739-748.

24. van der Kraan PM, van den Berg WB. Osteophytes: relevance and biology. Osteoarthritis Cartilage. 2007;15:237-244.

25. Wada M, Baba H, Imura S, Morita A, Kusaka Y. Relationship between radiographic classification and arthroscopic findings of articular cartilage lesions in osteoarthritis of the knee. Clin Exp Rheumatol. 1998;16:15-20.

26. Waldstein W, Bou Monsef J, Buckup J, Boettner F. The value of valgus stress radiographs in the workup for medial unicompartmental arthritis. Clin Orthop Relat Res. 2013;471:3998-4003.

27. Waldstein W, Merle C, Bou Monsef J, Boettner F. Varus knee osteoarthritis: how can we identify ACL insufficiency? Knee Surg Sports Traumatol Arthrosc. 2015;23:2178-2184.

28. Waldstein W, Perino G, Gilbert SL, Maher SA, Windhager R, Boettner F. OARSI osteoarthritis cartilage histopathology assessment system: a biomechanical evaluation in the human knee. J Orthop Res. 2016;34:135-140.

29. Waldstein W, Perino G, Jawetz ST, Gilbert SL, Boettner F. Does intraarticular inflammation predict biomechanical cartilage properties? Clin Orthop Relat Res. 2014;472:2177-2184. 